\title{
Deciphering the role of unique bacterial transcription-associated factor HelD.
}

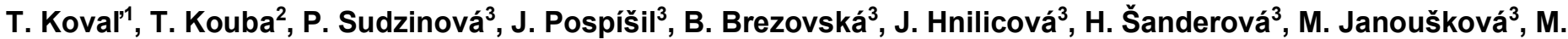 Šiková ${ }^{3}$, P. Halada ${ }^{3}$, M. Sýkora ${ }^{4}$, I. Barvík ${ }^{5}$, J. Nováček ${ }^{6}$, M. Trundová ${ }^{1}$, J. Dušková ${ }^{1}$, T. Skálová ${ }^{1}$, U. Chon ${ }^{7}$, K.S. Murakami $^{7}$, L. Krásný ${ }^{3}$, J. Dohnálek ${ }^{1}$}

${ }^{1}$ Institute of Biotechnology of the Czech Academy of Sciences, 25250 Vestec, Czech Republic, ${ }^{2}$ EMBL Grenoble, 71 Avenue des Martyrs, Grenoble, France, ${ }^{3}$ Institute of Microbiology of the Czech Academy of Sciences, Prague, Czech Republic, ${ }^{4}$ Institute of Molecular Genetics of the Czech Academy of Sciences, Prague, Czech Republic, ${ }^{5}$ Faculty of Mathematics and Physics, Institute of Physics, Charles University, Prague, Czech Republic, ${ }^{6}$ CEITEC, Masaryk University, Brno, Czech Republic, ${ }^{7}$ The Center for RNA Molecular Biology, Pennsylvania State University, University Park, PA 16802, USA)

tomas.koval@ibt.cas.cz

Bacterial RNA polymerase (RNAP) is an essential multisubunit enzyme performing transcription. Regulation of this process is secured through the stage-dependent interactions of RNAP with different factors (mostly proteins). Here we report the structurefunction analysis of the functional complexes between RNAP and a unique helicase-like factor HelD [1] which is present in many Gram-positive bacteria (e.g. Bacillus subtilis and Mycobacterium smegmatis) [2, 3]. HelD forms tightly bound complexes with RNAP. It simultaneously penetrates into RNAP primary and secondary channels which are responsible for nucleic acids binding and substrate delivery. HelD can also interact with the RNAP active site. Structurally, these interactions are incompatible with the binding of DNA to the RNAP core and thus with the elongation stage of transcription. This is in accordance to our functional data showing that HelD is capable of clearing RNAP of nucleic acids and that HelD can dismantle RNAP-DNA complexes. HelD itself is composed of several domains, showing structural changes in solution [2] as well as in complexes with RNAP (three different structural states obtained from the cryo-EM analysis) [3]. Although we were able to link the observed dynamic behaviour with the DNA-clearing role of HelD, the recycling of HelD-bound RNAP and subsequent restart of transcription remains to be explained.

HelD as well as its complexes with RNAP resisted our attempts to crystallize them for many years. In order to get to the structural details we took the advantage of recent developments in the field of single-particle cryo-EM and were able to obtain $\sim 3 \AA$ resolution structures. The structure of HelD itself was completely unknown with no homologue in the PDB. We combined X-ray crystallography (structure of one domain) and cryo-EM, together with bioinformatics and homologous modelling and successfully built de novo a complete atomic model of the HelD protein. For the analysis of condition-dependent dynamic behaviour we used small-angle X-ray scattering [2]. Results from our structural studies were supplemented with biochemical and biophysical assays (enzymology, analysis of interactions and stability) and by computational analyses [3].

[1] Wiedermannová, J., Sudzinová, P., Koval', T., Rabatinová, A., Šanderova, H., Ramaniuk, O., Rittich, Š. \& Dohnálek, J. (2014). Nucleic Acids Res. 42, 5151-5163.

[2] Koval', T., Sudzinová, P., Perháčová, T., Trundová, M., Skálová, T., Fejfarová, K., Šanderová, H., Krásný, L., Dušková, J. \& Dohnálek, J. (2019). FEBS Lett. 593, 996-1005.

[3] Kouba, T., Koval', T., Sudzinová, P., Pospíšil, J., Brezovská, B., Hnilicová, J., Šanderová, H., Janoušková, M., Šiková, M., Halada, P., Sýkora, M., Barvík, I., Nováček, J., Trundová, M., Dušková, J., Skálová, T., Chon, U., Murakami, K.S., Dohnálek, J. \& Krásný, L. (2020). Nat Commun.11, 6419.

Keywords: regulation of transcription; RNA polymerase, helicase-like factor HelD, structural changes

This work was supported by MEYS (LM2015043 and CZ.1.05/1.1.00/02.0109), CSF (20-12109S and 20-07473S), NIH (grant R35 GM131860), AS CR (86652036), ERDF (CZ.02.1.01/0.0/0.0/16_013/0001776 and CZ.02.1.01/0.0/0.0/15_003/0000447), EMBL (EI3POD) and Marie Skłodowska-Curie grant (664726). 